\title{
Behavioral and Neural Changes Induced by a Blended Essential Oil on Human Selective Attention
}

\author{
Jieqiong Liu, ${ }^{1}$ Shi Cai, ${ }^{2}$ Danni Chen, ${ }^{1}$ Ke Wu, ${ }^{2}$ Yang Liu, ${ }^{1}$ Ruqian Zhang, ${ }^{1}$ Mei Chen, ${ }^{1}$ \\ and Xianchun $\mathrm{Li} \mathbb{D}^{1}$ \\ ${ }^{1}$ School of Psychology and Cognitive Science, Shanghai Changning-ECNU Mental Health Center, East China Normal University, \\ Shanghai 200062, China \\ ${ }^{2}$ Daikin (China) Investment Co., Ltd., Shanghai 200062, China
}

Correspondence should be addressed to Xianchun Li; xcli@psy.ecnu.edu.cn

Received 4 March 2019; Accepted 7 August 2019; Published 15 October 2019

Academic Editor: Luigi Ferini-Strambi

Copyright (c) 2019 Jieqiong Liu et al. This is an open access article distributed under the Creative Commons Attribution License, which permits unrestricted use, distribution, and reproduction in any medium, provided the original work is properly cited.

\begin{abstract}
Selective attention refers to the selecting and preferential processing of specific information while simultaneously suppressing irrelevant distractors, activities linked to various cognitive skills and academic achievements. The influence of essential oils on the cognition of humans has been extensively explored. However, the effects of essential oils on human selective attention and the underlying neural mechanisms remain unclear. In the present study, participants were divided into a "blended essential oil" group and a "no essential oil" group and enrolled on a negative priming task, including a control condition and a negative priming condition. The event-related potential technique was used to examine the brain mechanisms underlying the blended essential oil effects on human selective attention. Behavioral results showed that individuals responded more quickly in the negative priming condition when exposed to the blended essential oil. In addition, the blended essential oil eliminated the differences in the P300 amplitude in the postcentral area of the brain between the negative priming condition and the control condition. Moreover, the blended essential oil led to stronger functional connectivity during the task. The present study thus suggests that blended essential oil can significantly change brain activity and functional connections in human beings, which may improve human selective attention.
\end{abstract}

\section{Introduction}

In daily life, we are presented with large amounts of information, but the capacity of the cognitive system is limited [1]. Therefore, it is essential for us to attend selectively to goal-relevant information while inhibiting distracting or irrelevant information. The mental process through which this is done is called selective attention. Many studies have demonstrated that performance on selective attention tasks is closely correlated with academic skills in general [2-4] and with specific cognitive abilities, including working memory [5], speech processing [6], and nonverbal intelligence [7]. Moreover, selective attention deficiency may be partially involved in certain neural disorders, such as autism spectrum disorder [8] and attention deficit disorder [9]. Therefore, it is important to take suitable measures to improve human selective attention.
Recently, essential oils have become highly popular in the field of alternative medicine. Since ancient times, both physical and mental disorders have been treated with essential oils, including rosemary [10], sage [11], and lavender [12]. Furthermore, many studies have suggested that the inhalation of essential oils improves mood, increases memory, and decreases stress [13-15]. It has been found that some essential oils, containing 1,8-cineole, menthol [16], $\beta$-pinene [17], and limonene [18], can have a stimulating effect on brain wave activity. One electroencephalography (EEG) study investigated the effect of the San-Jo-In essential oil on human neural activity. The results showed that during inhalation of the San-Jo-In essential oil, the EEG spectrum values of the fast alpha waves were enhanced by $50 \%$, suggesting that the essential oil was able to alter EEG activity and has positive effects on mood and the cognitive functions of the brain [19]. 
A handful of studies have demonstrated the effects of essential oils on human attention. For example, Shimizu et al. [20] tested the effects of essential oils on individuals' vigilance (referred to here as sustained attention). They found that the increase in reaction time was significantly lower with lavender than with the control, suggesting that lavender essential oil helps to restrain a decrease in vigilance or, in other words, to maintain sustained attention in longterm-vigilance tasks. It also confirmed the sedative effect of lavender on excessive arousal [20]. In another study, 20 older volunteers were randomly exposed to sage essential oil or a placebo, after which their memory and attention performance were measured using the Cognitive Drug Research computerized assessment system. Compared with the placebo condition, $333 \mathrm{mg}$ dose of sage essential oil significantly enhanced accuracy of attention and secondary memory performance [21]. In the current study, we selected a blended essential oil that mainly contained 1,8-cineole, 3carene, $\beta$-pinene, $\beta$-caryophyllene, and carnosic acid. The first four components can enhance the activation of the central nervous system (CNS) $[14,17,22]$, while carnosic acid can protect brain cells from oxidative damage and thereby improve memory and cognition [23]. Considering the positive effect of the above-mentioned components on both human attention and brain activities, we hypothesized that the blended essential oil should have a positive impact on human selective attention.

Negative priming (NP) is one of the most influential paradigms for measuring selective attention in cognitive psychology [24-26]. In the typical NP paradigm, a target and a distractor are displayed in the prime trial, followed by a similar probe trial. Participants are instructed to respond to the target and ignore the distractor. In such a task, NP refers to the difference in increased errors and response time (RT) between the probe target and the prime target. One frequently observed event-related potential (ERP) concerning visual identity NP is P300, which has been widely studied as a marker of attentional allocation and the updating of resources needed for stimulus evaluation in a variety of cognitive tasks [27, 28]. Some researchers have reported greater P300 amplitude in the NP condition than in the control condition in selective attention tasks $[29,30]$, implying that more attentional resources are engaged in NP trials. By contrast, lower amplitudes of $\mathrm{P} 1$ and $\mathrm{N} 1$ have been observed in the $\mathrm{NP}$ condition than in the control condition [31]. As the early sensory aspects of stimulus processing, the reduced amplitudes of $\mathrm{P} 1$ and N1 may indicate that sensory refractoriness is more pronounced for the control than for the NP condition. Previous findings have indicated that some brain regions are not only more activated but also more functionally connected when attentional processes are recruited [32].

In the present study, we investigated changes in selective attention induced by the blended essential oil and in related brain activity, using the ERP technique. We hypothesized that, at the behavioral level, the blended essential oil would decrease participants' RT in the NP condition. Correspondingly, at the neural level, P300 amplitude would be smaller in the frontal, parietal, and occipital areas in the same condition. Moreover, it was believed that interaction between distant brain areas enabled the flexible performance of cognitive tasks [33]. We speculated that intrabrain functional connectivity during the NP condition of the blended essential oil group is higher than that of the control group. Thus, the objective of this study was to use ERP to find out how the blended essential oil affected humans' performance on the selective attention task.

\section{Materials and Methods}

2.1. Participants. Forty-two undergraduates with a mean age of $21.3 \pm 2.7$ years $(M \pm S D)$ were recruited for the study. The participants were randomly assigned to the oil-treatment group or the control-treatment group by using a computergenerated list of pseudorandom integers between 1 and 2 (inclusive). Due to a misunderstanding of the task's requirements, head movements, and program malfunctions, there were 28 valid participants in total, 14 per group. All participants reported that they were in good health and did not have any preference or smell aversion to the blended essential oil, as determined by a pretest. Exclusion criteria included any history of brain diseases, psychiatric disorders, allergies to any essential oil or drug, cardiovascular disease, respiratory disease, or decrements in sense of smell. In addition, participants were asked to refrain from alcohol for a minimum of 12 hours and to have a good sleep of at least 6 hours prior to the experiment. Before the experiment, written informed consent was obtained from each participant. After completing the task, they received the corresponding remuneration. The study procedure was approved by the University Committee on Human Research Protection, East China Normal University.

2.2. Essential Oil Preparation. The blended essential oil, obtained from Daikin (China) Investment Co., Ltd. (Shanghai, China), was mainly composed of 1,8-cineole, 3-carene, $\beta$-pinene, $\beta$-caryophyllene, and carnosic acid. To maintain uniform concentrations of the blended essential oil throughout the room, several measures had to be taken before the experiment. The interval between the diffusion of the essential oil and the beginning of the task was about 30 minutes, which was enough for the blended essential oil to spread evenly around the room. The size of the EEG measurement room was $16 \mathrm{~m}^{2}$, with identical humidity $(50 \%-65 \%)$ and a constant temperature $\left(22^{\circ} \mathrm{C}\right)$. Two drops of the blended essential oil (about $0.1 \mathrm{ml}$ ) were added to fragrance tester strips and then diffused into the air by the special forced air systems provided by the Daikin (China) Investment Co., Ltd. These settings ensured that participants completed the task in an identical environment and that the blended essential oil was used to the best effect.

2.3. Stimuli. The stimuli were composed of letters and numbers. For the letter, the uppercase version of the consonants $\mathrm{M}, \mathrm{N}, \mathrm{P}, \mathrm{Q}, \mathrm{R}, \mathrm{T}$, and $\mathrm{X}$ was randomly selected. For the number, the digits $1,2,3,4,6,7$, and 8 were used. In each trial, the target stimulus appeared in the center of the screen and the distractor was randomly presented in one of four locations (over, under, left, or right) away from the center of the screen. 


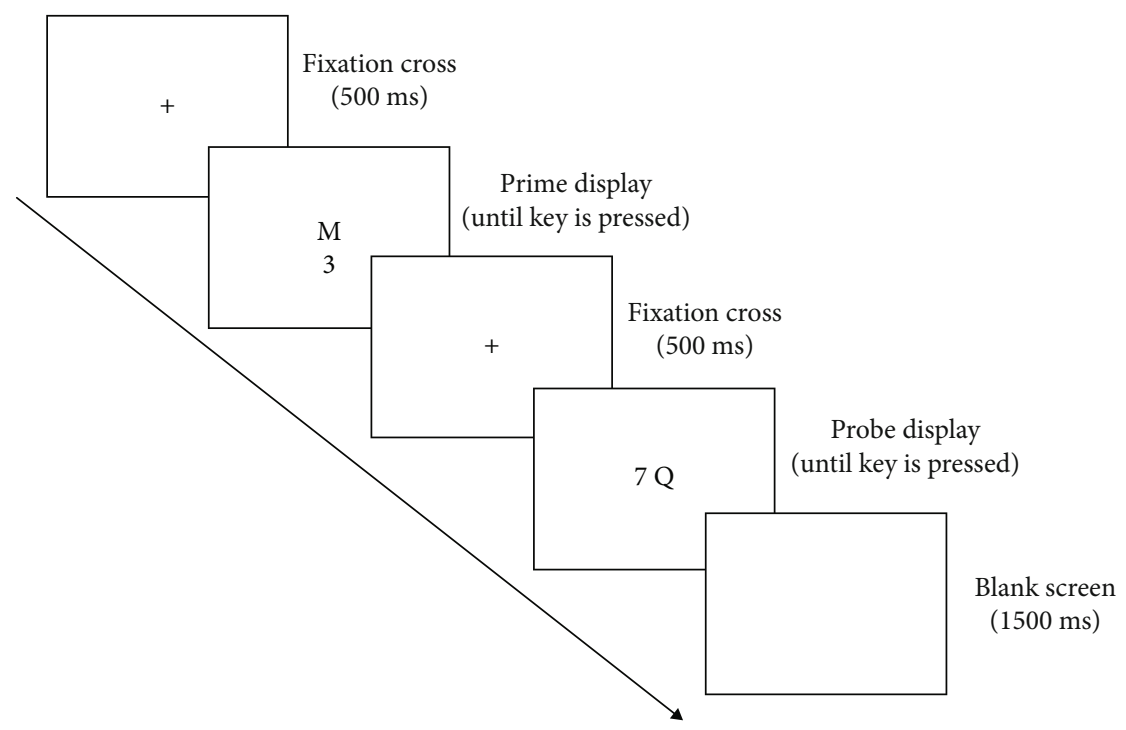

Figure 1: The experimental procedure.

The presentation of the stimuli and recording of the responses were controlled by E-prime software (Psychology Software Tools Inc. 2). All stimuli were presented on a high-resolution computer screen that was positioned at a distance of approximately $70 \mathrm{~cm}$ from the viewer.

2.4. Procedures. A practice session took place before the formal experiment. If the subjects had no questions about the experiment after the practice, they were able to start the experiment. Each trial began with a $500 \mathrm{~ms}$ fixation, followed by a presentation of the prime stimuli. Participants had to judge whether the target stimuli presented in the center of the screen were a letter or a number. If the response target was a letter, participants were required to press the " $F$ " key; if it was a number, they were to press the "J" key. After this, the same response was to be made for the probe stimuli.

Each trial consisted of prime stimuli and probe stimuli, resulting in four different types of combination: letter-letter, letter-number, number-number, and number-letter. The letter-number combination and number-letter combination were grouped as the NP condition. The letter-letter combination and number-number combination fell into the same category, namely, the positive priming condition, as the control condition. The task included 80 trials in total, 20 trials for each type, presented in a pseudorandom way (see Figure 1).

2.5. EEG Data Acquisition. EEGs were recorded using the Geodesic Photogrammetry System ${ }^{\mathrm{TM}}$ (GPS) with 64 channels (Electrical Geodesic Inc., USA). The electrode cap was in accordance with the international 10-10 system. We also recorded vertical and horizontal electrooculograms (EOGs) to control for eye movements and eye blinks. Electrode impedance was kept under $50 \mathrm{k} \Omega$ for all recordings. EEGs were continuously recorded at a sampling frequency of $250 \mathrm{~Hz}$.

2.6. EEG Data Analysis. EEG data analysis was conducted using EEGLAB [34] and custom MATLAB (MathWorks
Inc., Natick, MA) scripts. In the EEG data preprocessing, we applied a $0.1 \mathrm{~Hz}$ high-pass filter, a $30 \mathrm{~Hz}$ low-pass filter, and a $50 \mathrm{~Hz}$ notch filter consecutively to remove noise. EEG data were rereferenced using a common average reference. Then, to remove ocular artifacts (eye blinks and eye movements) and muscular artifacts, independent component analysis (ICA) was applied to the EEG data [34]; the ADJUST plug-in was then used to remove the ICA components that contained the artifacts [35]. EEG during each trial was segmented from $200 \mathrm{~ms}$ before the onset of the probe stimulus display to $500 \mathrm{~ms}$. Epochs were the baseline corrected to the $200 \mathrm{~ms}$ prestimulus baseline.

P300 amplitude was identified as the largest positive peak between 300 and $500 \mathrm{~ms}$ after the onset of the stimulus. We selected two electrodes of C3 and C4 in the frontal-central area, three electrodes of $\mathrm{Pz}, \mathrm{P} 3$, and $\mathrm{P} 4$ in parietal area, and three electrodes of $\mathrm{Oz}, \mathrm{O} 1$, and $\mathrm{O} 2$ in the occipital area for P300. A repeated measures ANOVA was performed on the mean P300 peak amplitude for probe stimuli with the priming condition as the within-subject variable (negative prime or control) and treatment (essential oils or no essential oils) as the between-subject variable. The false discovery rate (FDR) correction was conducted on the $p$ values of the interaction effect to avoid a "Type I" error.

2.7. Intrabrain Functional Connectivity Analysis. Synchronization is usually measured between two signals across time and used to measure intra-/interbrain functional connectivity. The EEG dynamics was then quantified with the timevarying phase-locking value (PLV) which provided an index of neural synchrony. After a band pass filtering of the EEG signal, we applied a Hilbert transform to calculate the instantaneous phase $\phi$ of each electrode signal. The PLV formula for two channels (such as $p$ and $q$ ) is given by

$$
\operatorname{PLV}_{p, q}=\frac{1}{T}\left|\sum_{t=1}^{T} \exp ^{i\left(\phi p^{(t)}-\phi q^{(t)}\right)}\right|
$$


This formula was calculated across all epochs of one subject, with $T$ representing the number of trials. Four frequency bands were chosen for analysis: theta band (4-7 Hz), alpha band $(8-12 \mathrm{~Hz})$, beta band $(13-30 \mathrm{~Hz})$, and gamma band (31-48 Hz). The intrabrain functional connectivity was characterized by an increase of the average PLV values in the anatomical coupling on the oscillatory activity among electrodes. All PLV values among electrode pairs were subjected to a $t$-test and were corrected using the false discovery rate $(p<0.05$, FDR correction) [36].

\section{Results}

3.1. Behavioral Results. Trials containing incorrect responses or those with response times (RTs) deviated more than two standard deviations from an individual's mean RT were excluded for further analysis. The results of a two-way ANOVA on response time showed a main effect of priming condition (see Figure 2, $F(1,26)=17.89, p<0.001, \eta^{2}=$ $0.41)$. The response time in the negative priming condition was significantly longer compared with the control condition. These results indicated a reliable negative priming effect. A significant difference was also found for the main effect of the treatment (see Figure 2, $F(1,26)=4.80, p=$ $\left.0.038, \eta^{2}=0.16\right)$. The blended essential oil group showed a shorter response time compared to the no essential oil group.

\subsection{EEG Results}

3.2.1. P300 in the Frontal-Central Area. A repeated-measures ANOVA, used to examine the mean P300 in the frontalcentral area, revealed a significant interaction effect of the priming condition and the treatment at C4 (see Figure 3(a), $F(1,26)=6.34, p=0.04$, FDR correction, $\left.\eta^{2}=0.20\right)$. The simple effects analysis showed that the mean P300 amplitude of the negative priming trials was greater than that of control trials for the group without the blended essential oil (see Figure 3(b), $t(13)=2.18, p=0.048$, FDR correction, Cohen's $d=0.68$ ), whereas there was no such effect in the oil-treatment group. The interaction of treatment by priming condition did not reach a significant level at C3 $(p>0.05$, FDR correction).

3.2.2. $P 300$ in the Parietal Area. Similarly, a repeatedmeasures ANOVA analysis of the mean P300 also revealed a significant interaction effect at P3 in the parietal area (see Figure $3(\mathrm{c}), F(1,26)=6.82, p=0.04$, FDR correction, $\eta^{2}=$ $0.21)$. The simple effects analysis showed that the negative priming condition induced greater P300 compared to the control condition in the no essential oil group (see Figure $3(\mathrm{~d}), t(13)=2.96, p=0.01$, FDR correction, Cohen's $d=0.69)$. However, no such difference was observed in the blended essential oil group. No interaction effect was observed at $\mathrm{Pz}$ or $\mathrm{P} 4$ ( $p>0.05$, FDR correction).

3.2.3. P300 in the Occipital Area. The same ANOVA analysis showed a significant interaction effect of priming type and treatment on P300 at $\mathrm{Oz}$ (see Figure $3(\mathrm{e}), F(1,26)=6.77$, $p=0.04$, FDR correction, $\left.\eta^{2}=0.21\right)$. In detail, the mean

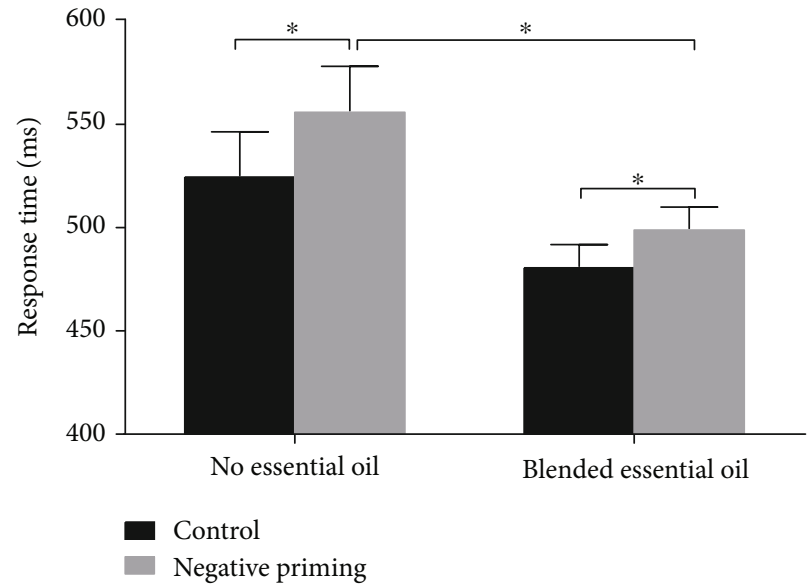

Figure 2: Behavioral results. The mean response times (RTs) of two priming conditions (control and negative priming) for different groups (no essential oil and blended essential oil). ${ }^{*} p<0.05$.

P300 amplitude of the negative priming trials was greater than control trials in the group without the blended essential oil (see Figure 3(f), $t(13)=2.79, p<0.048$, FDR correction, Cohen' s $d=0.93$ ), but not in the blended essential oil group. Nevertheless, no significant interaction effect was found at O1 or $\mathrm{O} 2(p>0.05$, FDR correction).

3.3. Intrabrain Functional Connectivity. The EEG data was then analyzed with the Phase Locking Value (PLV) which provided a measurement to quantify functional connectivity between two areas in the brain. The PLV analysis showed that essential oils induced stronger intrabrain functional connections during performance of the selective attention task. Specifically, higher PLVs in alpha band were found in $\mathrm{Pz}-\mathrm{Fz}(t(13)=-4.05, p=0.01$, Cohen's $d=1.53)$, Pz-F4 $(t(13)=-4.20, p=0.02$, Cohen's $d=1.59)$, and C4$\mathrm{P} 4(t(13)=-3.56, p=0.03$, Cohen's $d=1.35)$ in the blended essential oil group compared to the no essential oil group (see Figures 4(a) and 4(c)).

Meanwhile, increased PLVs in the beta band were observed within the Pz-F4 $(t(13)=-4.23, p=0.01$, Cohen's $d=1.60)$ and Oz-F4 $(t(13)=-4.20, p=0.01$, Cohen's $d=$ 1.59) (see Figures 4(b) and 4(d)) in the blended essential oil group compared to that in the no essential oil group. Thus, the blended essential oil could strengthen functional connectivity between the frontal lobe and parietal lobe in both alpha band $(\mathrm{Pz}-\mathrm{Fz}, \mathrm{Pz}-\mathrm{F} 4$, and $\mathrm{P} 4-\mathrm{C} 4)$ and beta band $(\mathrm{Pz}-\mathrm{F} 4$ and $\mathrm{Oz}-\mathrm{F} 4)$.

\section{Discussion}

In the present study, we used the ERP technique to examine the effect of blended essential oil on human selective attention and the neural basis of this effect. Behavioral results showed that the blended essential oil was able to improve participants' performance in the NP task by shortening RT in the NP trials. ERP results revealed the interaction effect of the treatment and the priming condition. The P300 amplitudes at $\mathrm{C} 4, \mathrm{P} 3$, and $\mathrm{Oz}$ were significantly higher in the NP condition than in the control condition for the "no 


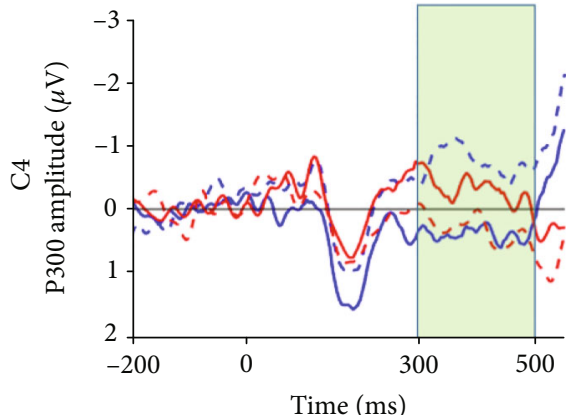

(a)

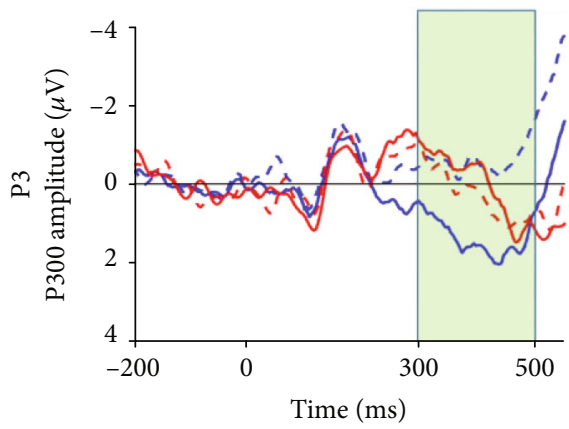

(c)

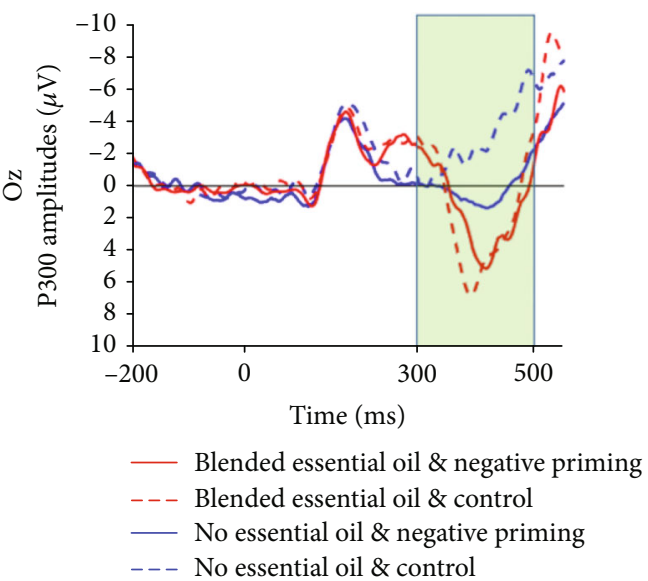

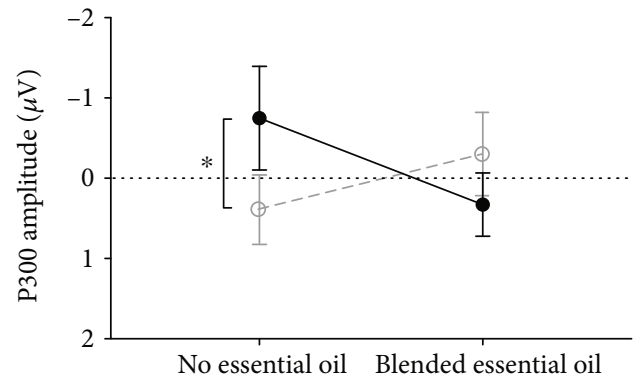

(b)

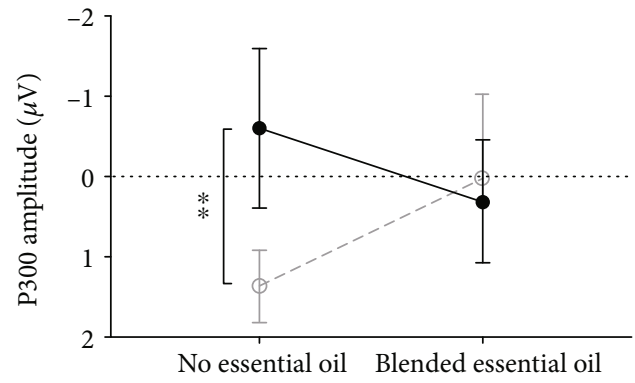

(d)

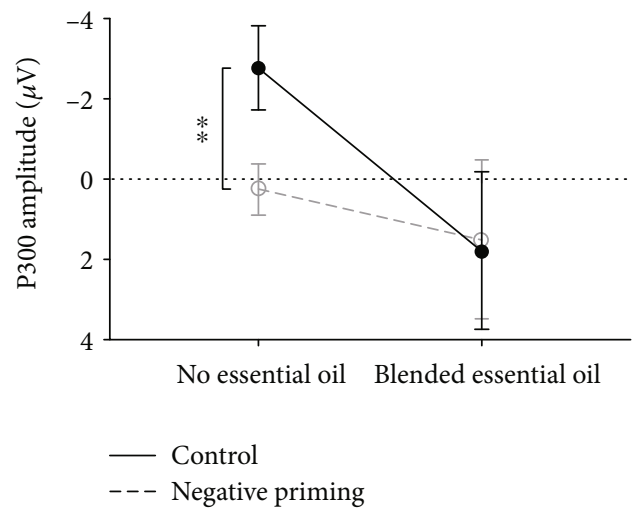

(f)

(e)

Figure 3: P300 waveforms and mean P300 amplitudes. (a, b) The amplitude in the time window 300-500 ms after the probe stimulus onset for the P300 component at C4. (c, d) The amplitude in the time window 300-500 ms after the probe stimulus onset for the P300 component at P3. (e, f) The amplitude in the time window 300-500 ms after the probe stimulus onset for the P300 component at Oz. ${ }^{*} p<0.05$ and ${ }^{* *} p$ $<0.01$.

essential oil" group. However, this effect disappeared when participants were exposed to the blended essential oil. A PLV analysis showed stronger intrabrain synchrony in the alpha band (Pz-Fz, Pz-F4, and P4-C4) and beta band (Pz$\mathrm{F} 4$ and $\mathrm{Oz}-\mathrm{F} 4)$ in the oil-treatment group than in the "no essential oil" group. Our findings provided both behavioral and neural evidence for the positive effect of blended essential oil on human selective attention.

First, the behavioral results showed that the mean RT of the NP condition was longer than that of the control condition in both groups, demonstrating a NP effect consistent with previous works [37-39]. The quicker response for the
NP trials in the blended essential oil group reflected better performance in selective attention. Together, these findings support our hypothesis that inhalation of the blended essential oil can improve selective attention.

Second, as reported in previous studies [37, 40], the NP effect was accompanied by an increase in P300 amplitude in the "no essential oil" group. However, the same result was not found in the oil-treatment group. P300 amplitude is often interpreted as an index of updating resources and mental efforts that are needed for stimulus in a variety of cognitive tasks [41]. Thus, it is generally assumed that the larger P300 amplitude corresponds to a greater allocation of 


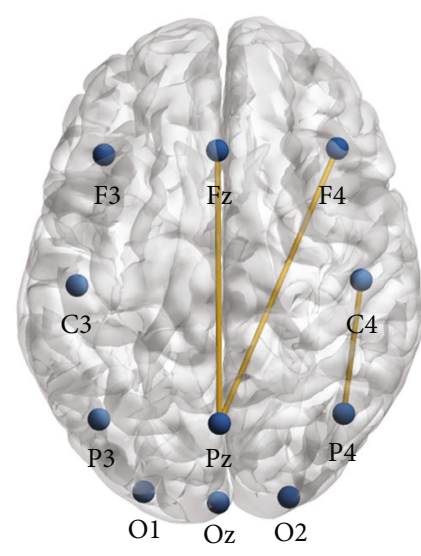

(a)

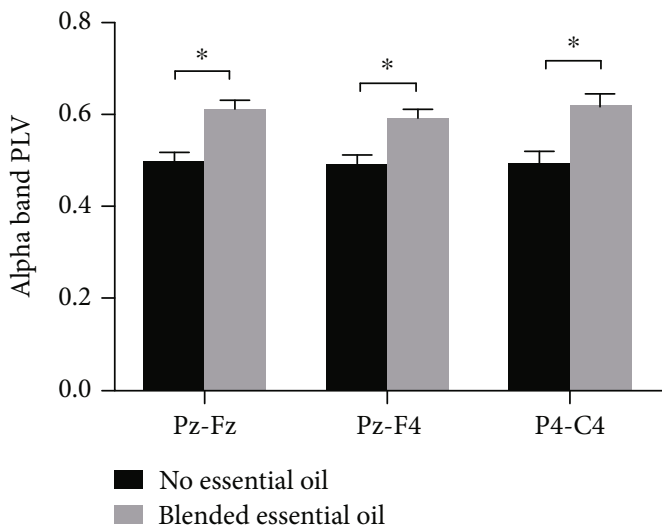

(c)

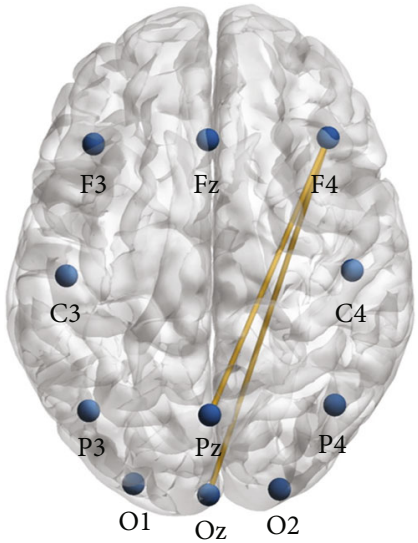

(b)

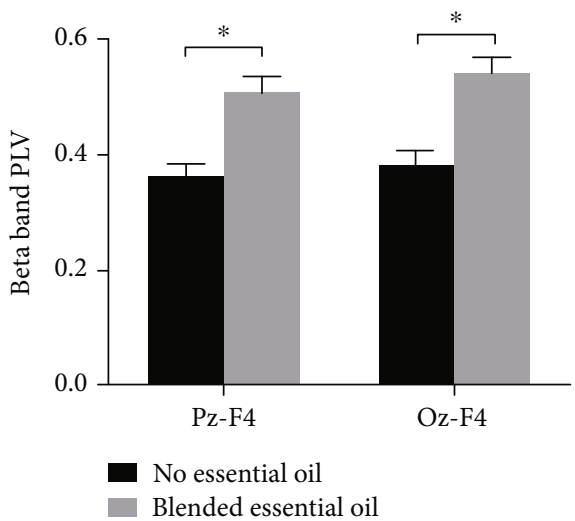

(d)

FIgURE 4: Intrabrain functional connectivity. (a) The increased intrabrain PLVs in alpha band in the blended essential oil group compared to the no essential oil group. (b) The increased intrabrain PLVs in beta band in the blended essential oil group compared to the no essential oil group. (c) The comparison of mean alpha band intrabrain PLVs in Pz-Fz, Pz-F4, and P4-C4. (d) The comparison of mean beta band intrabrain PLVs in Pz-F4 and Oz-F4. ${ }^{*} p<0.05$, FDR correction.

attention. Our ERP findings therefore suggest the neural mechanism through which the blended essential oil influenced human selective attention. That is, the blended essential oil was able to facilitate processing and decrease the mental effort in the NP by affecting P300 amplitude.

Third, the inhalation of blended essential oil can significantly increase the PLV in the alpha and beta bands, which resembled the behavioral pattern of accuracy very well. The PLV provided a frequency-specific synchronization measure between two signals across time [42] and interaction between neural assemblies [43]. It is believed that this interaction between distant brain areas may promote the formation exchange of transient functional networks, enabling the flexible performance of cognitive tasks $[33,43,44]$. This finding was also consonant with previous ERP studies indicating that increased phase locking between the parietal cortex and occipital cortex during attention is associated with enhanced performance [45]. Thus, the greater intrabrain alpha and beta band synchronization in the blended essential oil group may be relevant for the successful engagement of selective attention.

The results of this study show that exposure to the aroma of a blended essential oil can significantly enhance performance on NP tasks in humans by modulating brain wave activity and brain functional connections. Our findings partly support prior observations that some essential oils significantly improve the psychophysiological conditions of humans, such as attention, working capacity, mood, and stress. The main chemical components of the blended essential oil used in this experiment are 1,8-cineole, 3-carene, $\beta$-pinene, and $\beta$-caryophyllene, which have been proven to have a stimulating effect on sympathetic activity. It may be beneficial for the brain to remain active and vigilant [46], which may enhance the cognitive performance of humans. In addition, an increase of reactive oxygen species (ROS) in the brain tissues of humans can lead to neurotoxicity damage and oxidative stress [47]. Carnosic acid and 1,8cineole can attenuate oxidative injury by inhibiting ROS production and increasing endogenous antioxidant compounds $[48,49]$. The present findings indicate that the blended essential oil's effects on task performance are mainly associated with the stimulation of sympathetic activity and subsequent alteration of brain activity and function.

Our study is not without limitations. First, it was difficult for us to identify the precise brain regions for selective attention because of the low spatial resolution of the EEG 
technique. Future research could investigate the neural mechanisms underlying selective attention by combining fMRI and EEG techniques. Second, PLV has been challenged for its spurious hyperconnections [50]. Measures and analyses that are more robust should be applied in future studies to consolidate the current findings.

In summary, the blended essential oil appears to improve performance in selective attention tasks by shortening response times in NP trials, modulating P300 amplitude, and increasing intrabrain phase synchronization in the frontal-parietal networks. Our findings suggest that the blended essential oil could be used as a stimulant to improve human selective attention.

\section{Conclusions}

In summary, the blended essential oil appears to improve performance in selective attention tasks by shortening response times in NP trials, modulating P300 amplitude, and increasing intrabrain phase synchronization in the frontal-parietal networks. Our findings suggest that the blended essential oil could be used as a stimulant to improve human selective attention.

\section{Data Availability}

The data used to support the findings of this study are available from the corresponding author upon request.

\section{Conflicts of Interest}

Authors Shi Cai and Ke Wu were employed by the company Daikin (China) Investment Co., Ltd., Shanghai, China 200062. All other authors declare that there is no conflict of interest regarding the publication of this paper.

\section{Acknowledgments}

The authors thank Di Yuan for her valuable help and comments in earlier drafts of the paper. This work was supported by the East China Normal University Academic Innovation Promotion Program for Excellent Doctoral Students (YBNLTS2019-024).

\section{References}

[1] M. I. Posner, C. R. Snyder, and R. Solso, "Attention and cognitive control," in Cognitive Psychology: Key Readings, pp. 205223, Psychology Press, New York, NY, USA, 2004.

[2] E. Commodari and M. Di Blasi, "The role of the different components of attention on calculation skill," Learning and Individual Differences, vol. 32, pp. 225-232, 2014.

[3] A. Steele, A. Karmiloff-Smith, K. Cornish, and G. Scerif, "The multiple subfunctions of attention: differential developmental gateways to literacy and numeracy," Child Development, vol. 83, no. 6, pp. 2028-2041, 2012.

[4] C. Stevens and D. Bavelier, "The role of selective attention on academic foundations: a cognitive neuroscience perspective," Developmental Cognitive Neuroscience, vol. 2, Supplement 1, pp. S30-S48, 2012.
[5] R. J. Giuliano, C. M. Karns, H. J. Neville, and S. A. Hillyard, "Early auditory evoked potential is modulated by selective attention and related to individual differences in visual working memory capacity," Journal of Cognitive Neuroscience, vol. 26, no. 12, pp. 2682-2690, 2014.

[6] L. B. Astheimer and L. D. Sanders, "Temporally selective attention supports speech processing in 3- to 5-year-old children," Developmental Cognitive Neuroscience, vol. 2, no. 1, pp. 120 128, 2012.

[7] E. Isbell, A. H. Wray, and H. J. Neville, "Individual differences in neural mechanisms of selective auditory attention in preschoolers from lower socioeconomic status backgrounds: an event-related potentials study," Developmental Science, vol. 19, no. 6, pp. 865-880, 2016.

[8] K. Markram and H. Markram, "The intense world theory - a unifying theory of the neurobiology of autism," Frontiers in Human Neuroscience, vol. 4, p. 224, 2010.

[9] C. L. Armstrong, K. M. Hayes, and R. Martin, "Neurocognitive problems in attention deficit disorder," Annals of the New York Academy of Sciences, vol. 931, no. 1, pp. 196215, 2001.

[10] W. Sayorwan, N. Ruangrungsi, T. Piriyapunyporn, T. Hongratanaworakit, N. Kotchabhakdi, and V. Siripornpanich, "Effects of inhaled rosemary oil on subjective feelings and activities of the nervous system," Scientia Pharmaceutica, vol. 81, no. 2, pp. 531-542, 2012.

[11] D. O. Kennedy, F. L. Dodd, B. C. Robertson et al., "Monoterpenoid extract of sage (Salvia lavandulaefolia) with cholinesterase inhibiting properties improves cognitive performance and mood in healthy adults," Journal of Psychopharmacology, vol. 25 , no. 8, pp. 1088-1100, 2011.

[12] W. Dimpfel, I. Pischel, and R. Lehnfeld, "Effects of lozenge containing lavender oil, extracts from hops, lemon balm and oat on electrical brain activity of volunteers," European Journal of Medical Research, vol. 9, no. 9, pp. 423-431, 2004.

[13] J. Lehrner, G. Marwinski, S. Lehr, P. Johren, and L. Deecke, "Ambient odors of orange and lavender reduce anxiety and improve mood in a dental office," Physiology \& Behavior, vol. 86, no. 1-2, pp. 92-95, 2005.

[14] M. Moss, J. Cook, K. Wesnes, and P. Duckett, “Aromas of rosemary and lavender essential oils differentially affect cognition and mood in healthy adults," International Journal of Neuroscience, vol. 113, no. 1, pp. 15-38, 2003.

[15] A. Bikmoradi, Z. Seifi, J. Poorolajal, M. Araghchian, R. Safiaryan, and K. Oshvandi, "Effect of inhalation aromatherapy with lavender essential oil on stress and vital signs in patients undergoing coronary artery bypass surgery: a singleblinded randomized clinical trial," Complementary Therapies in Medicine, vol. 23, no. 3, pp. 331-338, 2015.

[16] T. Umezu, A. Sakata, and H. Ito, “Ambulation-promoting effect of peppermint oil and identification of its active constituents," Pharmacology Biochemistry and Behavior, vol. 69, no. 3-4, pp. 383-390, 2001.

[17] S. U. Savelev, E. J. Okello, and E. K. Perry, "Butyryl- and acetylcholinesterase inhibitory activities in essential oils of Salvia species and their constituents," Phytotherapy Research, vol. 18, no. 4, pp. 315-324, 2004.

[18] E. Heuberger, T. Hongratanaworakit, C. Böhm, R. Weber, and G. Buchbauer, "Effects of chiral fragrances on human autonomic nervous system parameters and self-evaluation," Chemical Senses, vol. 26, no. 3, pp. 281-292, 2001. 
[19] H. Cho, B. Yu, K. Sowndhararajan, J.-W. Jung, J.-W. Jhoo, and S. Kim, "Effect of essential oil from San-Jo-In (Zizyphus jujuba Mill. seeds) on human electroencephalographic activity," Journal of Life Science, vol. 23, no. 9, pp. 11701176, 2013.

[20] K. Shimizu, M. Gyokusen, S. Kitamura et al., "Essential oil of lavender inhibited the decreased attention during a longterm task in humans," Bioscience, Biotechnology, and Biochemistry, vol. 72, no. 7, pp. 1944-1947, 2008.

[21] A. B. Scholey, N. T. J. Tildesley, C. G. Ballard et al., "An extract of Salvia (sage) with anticholinesterase properties improves memory and attention in healthy older volunteers," Psychopharmacology, vol. 198, no. 1, pp. 127-139, 2008.

[22] J. P. Royet, J. Hudry, D. H. Zald et al., "Functional neuroanatomy of different olfactory judgments," Neuroimage, vol. 13, no. 3, pp. 506-519, 2001.

[23] T. Satoh, K. Kosaka, K. Itoh et al., "Carnosic acid, a catecholtype electrophilic compound, protects neurons both in vitro and in vivo through activation of the Keap1/Nrf2 pathway via $S$-alkylation of targeted cysteines on Keap1," Journal of Neurochemistry, vol. 104, no. 4, pp. 1116-1131, 2008.

[24] M. T. Daza, J. J. Ortells, and C. Noguera, "Negative semantic priming from consciously vs. unconsciously perceived single words," Psicológica, vol. 28, no. 2, pp. 105-127, 2007.

[25] L. Machado, H. Guiney, and P. Struthers, "Identity-based inhibitory processing during focused attention," The Quarterly Journal of Experimental Psychology, vol. 66, no. 1, pp. 138-159, 2013.

[26] J. J. Ortells, C. Noguera, D. Álvarez, E. Carmona, and G. Houghton, "Individual differences in working memory capacity modulates semantic negative priming from single prime words," Frontiers in Psychology, vol. 7, article 1286, 2016.

[27] J. Polich, "Updating P300: an integrative theory of P3a and P3b," Clinical Neurophysiology, vol. 118, no. 10, pp. $2128-$ 2148, 2007.

[28] L. M. Riby, S. I. Sünram-Lea, C. Graham et al., "P3b versus P3a: an event-related potential investigation of the glucose facilitation effect," Journal of Psychopharmacology, vol. 22, no. 5, pp. 486-492, 2008.

[29] F. Pan, L. Shi, Q. Lu, X. Wu, S. Xue, and Q. Li, "The negative priming effect in cognitive conflict processing," Neuroscience letters, vol. 628, pp. 35-39, 2016.

[30] C. Frings and C. Groh-Bordin, "Electrophysiological correlates of visual identity negative priming," Brain Research, vol. 1176, pp. 82-91, 2007.

[31] H. Gibbons, T. H. Rammsayer, and J. Stahl, "Multiple sources of positive- and negative-priming effects: an event-related potential study," Memory \& Cognition, vol. 34, no. 1, pp. 172-186, 2006.

[32] L. Wang, X. Liu, K. G. Guise, R. T. Knight, J. Ghajar, and J. Fan, "Effective connectivity of the fronto-parietal network during attentional control," Journal of Cognitive Neuroscience, vol. 22, no. 3, pp. 543-553, 2010.

[33] W. Klimesch, P. Sauseng, and S. Hanslmayr, "EEG alpha oscillations: the inhibition-timing hypothesis," Brain Research Reviews, vol. 53, no. 1, pp. 63-88, 2007.

[34] A. Delorme and S. Makeig, "EEGLAB: an open source toolbox for analysis of single-trial EEG dynamics including independent component analysis," Journal of Neuroscience Methods, vol. 134, no. 1, pp. 9-21, 2004.
[35] A. Mognon, J. Jovicich, L. Bruzzone, and M. Buiatti, “ADJUST: an automatic EEG artifact detector based on the joint use of spatial and temporal features," Psychophysiology, vol. 48, no. 2, pp. 229-240, 2011.

[36] Y. Benjamini and Y. Hochberg, "Controlling the false discovery rate: a practical and powerful approach to multiple testing," Journal of the Royal Statistical Society: Series B (Methodological), vol. 57, no. 1, pp. 289-300, 1995.

[37] N. Kathmann, B. Bogdahn, and T. Endrass, "Event-related brain potential variations during location and identity negative priming," Neuroscience Letters, vol. 394, no. 1, pp. 53-56, 2006.

[38] H. Gibbons, "Functional brain-electrical correlates of negative priming in the flanker task: evidence for episodic retrieval," Psychophysiology, vol. 46, no. 4, pp. 807-817, 2009.

[39] J. Stahl and H. Gibbons, "Event-related brain potentials support episodic-retrieval explanations of flanker negative priming," Experimental Brain Research, vol. 181, no. 4, pp. 595-606, 2007.

[40] S. Watanabe, K. Hara, K. Ohta et al., “Aroma helps to preserve information processing resources of the brain in healthy subjects but not in temporal lobe epilepsy," Seizure, vol. 22, no. 1, pp. 59-63, 2013.

[41] E. Donchin and M. G. Coles, "Is the P300 component a manifestation of context updating?," Behavioral and Brain Sciences, vol. 11, no. 3, pp. 357-374, 1988.

[42] J. P. Lachaux, E. Rodriguez, J. Martinerie, and F. J. Varela, "Measuring phase synchrony in brain signals," Human Brain Mapping, vol. 8, no. 4, pp. 194-208, 1999.

[43] P. Fries, "A mechanism for cognitive dynamics: neuronal communication through neuronal coherence," Trends in Cognitive Sciences, vol. 9, no. 10, pp. 474-480, 2005.

[44] F. Varela, J.-P. Lachaux, E. Rodriguez, and J. Martinerie, "The brainweb: phase synchronization and large-scale integration," Nature Reviews Neuroscience, vol. 2, no. 4, p. 229, 2001.

[45] S. M. Doesburg, J. J. Green, J. J. McDonald, and L. M. Ward, "From local inhibition to long-range integration: a functional dissociation of alpha-band synchronization across cortical scales in visuospatial attention," Brain Research, vol. 1303, pp. 97-110, 2009.

[46] M. Kim, K. Sowndhararajan, S. J. Park, and S. Kim, "Effect of inhalation of isomers, $(+)-\alpha$-pinene and $(+)-\beta$-pinene on human electroencephalographic activity according to gender difference," European Journal of Integrative Medicine, vol. 17, pp. 33-39, 2018.

[47] M. T. Islam, "Oxidative stress and mitochondrial dysfunctionlinked neurodegenerative disorders," Neurological Research, vol. 39, no. 1, pp. 73-82, 2017.

[48] M. Porres-Martínez, E. González-Burgos, M. E. Carretero, and M. P. Gómez-Serranillos, "Major selected monoterpenes $\alpha$-pinene and 1,8-cineole found in Salvia lavandulifolia (Spanish sage) essential oil as regulators of cellular redox balance," Pharmaceutical Biology, vol. 53, no. 6, pp. 921929, 2014.

[49] D. M. Miller, I. N. Singh, J. A. Wang, and E. D. Hall, "Nrf2ARE activator carnosic acid decreases mitochondrial dysfunction, oxidative damage and neuronal cytoskeletal degradation following traumatic brain injury in mice," Experimental Neurology, vol. 264, pp. 103-110, 2015.

[50] A. P. Burgess, "On the interpretation of synchronization in EEG hyperscanning studies: a cautionary note," Frontiers in Human Neuroscience, vol. 7, p. 881, 2013. 


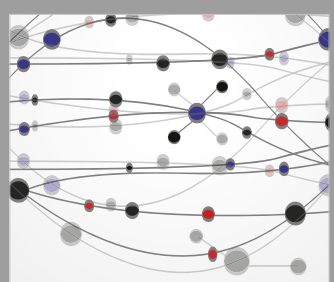

The Scientific World Journal
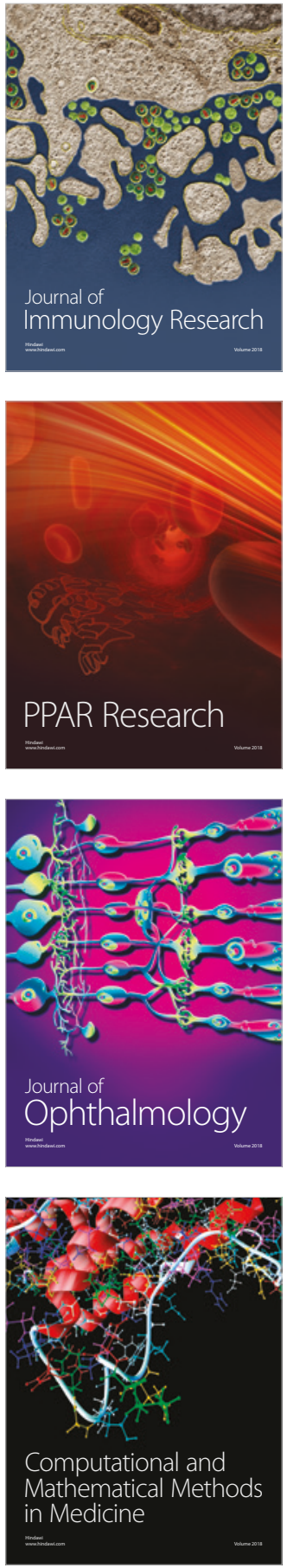

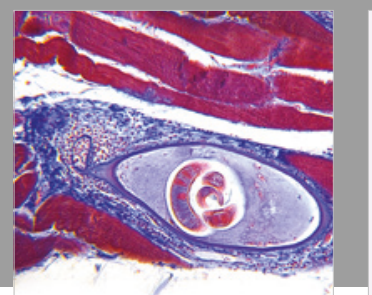

Gastroenterology Research and Practice

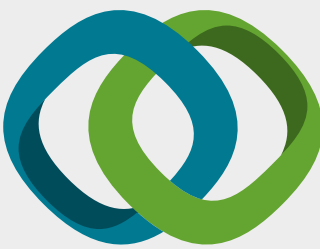

\section{Hindawi}

Submit your manuscripts at

www.hindawi.com
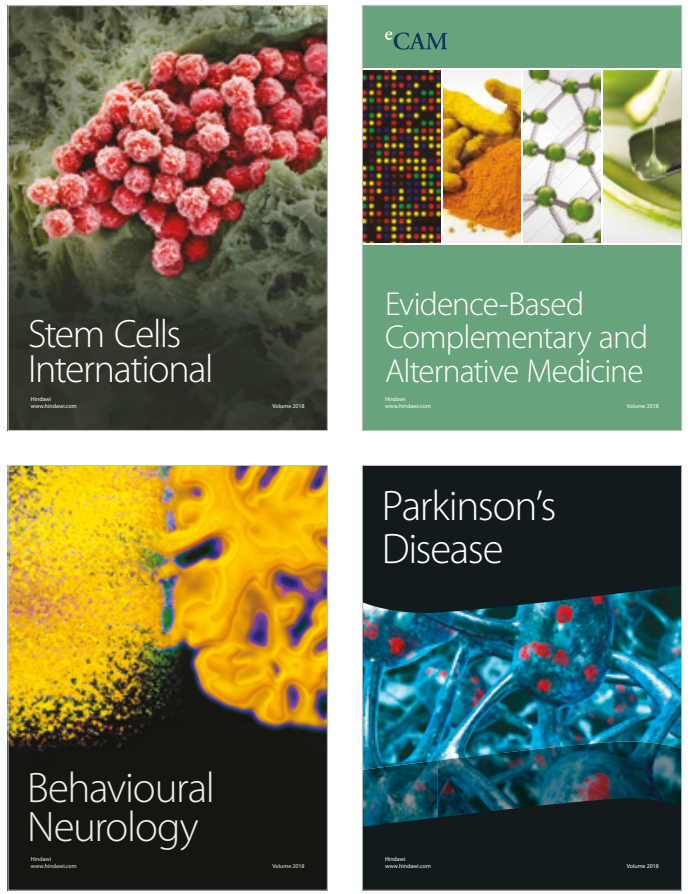

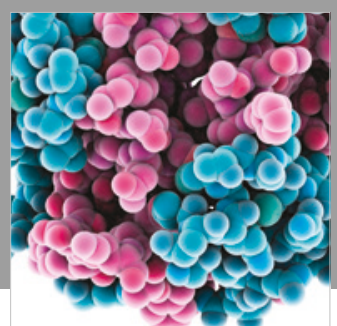

ournal of

Diabetes Research

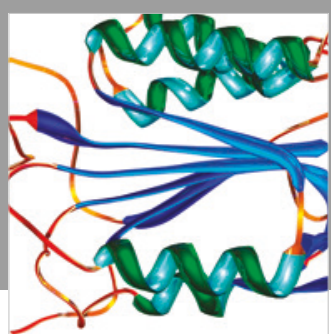

Disease Markers
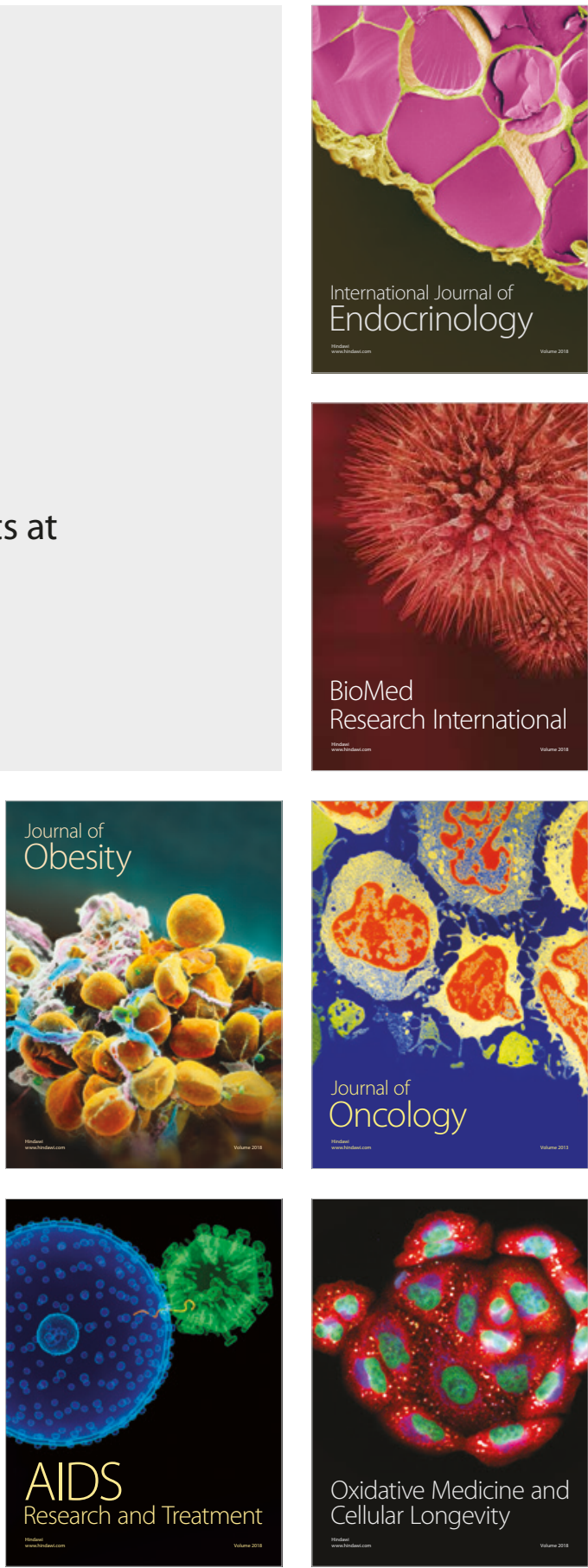